\title{
AMELIE: Authoring Multimedia-Enhanced Learning Interactive Environment for e-Health Contents
}

\author{
P. Sánchez-González I. Oropesa P. Moreno-Sánchez \\ J.M. Martínez-Moreno J. García-Novoa and E.J. Gómez
}

\begin{abstract}
This paper presents the AMELIE Authoring Tool for e-health applications. AMELIE provides the means for creating video-based contents with a focus on e-learning and telerehabilitation processes. The main core of AMELIE lies in the efficient exploitation of raw multimedia resources, which may be already available at clinical centers or recorded ad hoc for learning purposes by health professionals. Three real use cases scenarios involving different target users are presented: (1) cognitive skills' training of surgeons in minimally invasive surgery (medical professionals), (2) training of informal carers for elderly home assistance and (3) cognitive rehabilitation of patients with acquired brain injury. Preliminary validation in the field of surgery hints at the potential of AMELIE; and its versatility in different medical applications is patent from the use cases described. Regardless, new validation studies are planned in the three main application areas identified in this work.
\end{abstract}

Keywords-Medical education, e-learning, telerehabilitation, AMELIE, authoring tool.

\section{INTRODUCTION}

The evolution of technology-enhanced learning (TEL) has brought forth a new education paradigm where cost, time and geographical barriers are no longer a constraining issue. Due to its nature, it provides the necessary learning conditions anytime and anywhere, as well as personalize the learner's experience and formative paths without compromising the consistency of training programs [1].

All advantages considered, TEL poses an opportunity to ease formative requirements in health applications at all levels: from the training and assessment of new professionals (doctors, nurses) to the instruction of informal carers or the rehabilitation of neural injuries.

A critical aspect in the definition of TEL environments resides in the quality and appeal of the provided didactic contents. It has been pointed out that these contents should promote interactivity with the learner rather than be simple passive units [2]. Multimedia resources, especially in the form of procedural videos, are often available at hospitals and clinical centers. While they may be used for classroom tuition, more often than not they are not fully exploited.
Moreover, the creation of these contents can be tedious, counterintuitive and difficult [3].

In this paper, we present AMELIE (Authoring Multimedia-Enhanced Learning Interactive Environment), an authoring tool for the creation of multimedia didactic contents in clinical applications by health professionals. The tool contemplates the definition of complete, personalized didactic units focused on the exploitation of augmented video resources, with an emphasis on reusability and sharing of contents. The work also presents three real medical use cases where the authoring tool is currently being applied, addressing the different actors involved in the clinical system (medical professionals, informal carers and patients).

\section{AUTHORING TOOL}

The main core of AMELIE lies in the efficient exploitation of raw multimedia resources for video-based learning and telerehabilitation, which may be already available at hospitals (e.g.: surgical interventions, 4D image studies, etc.) or recorded ad hoc for learning purposes by health professionals. Didactic units may be created, structured in an indeterminate number of sections (determined by the author), including text, image and enhanced videos.

For every section the content creator wishes to include, he/she may add a new tab to the didactic unit. Two main content tabs can be added: (1) regular and (2) video-based. In the first, space is provided to include a main text description, along with images and any additional relevant files. The latter allows the inclusion of an augmented video resource, and provides direct access to an editor where the author may process the raw video (Fig. 1).

The editor offers intuitive methods to modify a video resource by: (1) adding text, audio or image overlays at different points of the recording; (2) crop the video; (3) modify its visual properties (contrast, brightness, etc.); (4) create clips; (5) document the videos; (6) include assessment breakpoints, where the student must answer questions on the video before moving on; and (7) tag and track objects on the screen (by means of segmentation and tracking algorithms specifically designed to that end). 
Finally, the possibility of creating an assessment tab is provided. Available functionalities allow for the definition, edition and deletion of evaluation questions (multiple choice/sorting test-type questions). Additionally, the creator may choose where to place said questions, whether it be within a specific section or on a whole new one at the end of the didactic unit.

A special video player is provided to account for augmented features of the video resources. It allows playback of the different text/audio/assessment/segmentation overlays, as well as the possibility of toggling them on/off according to users' preferences.

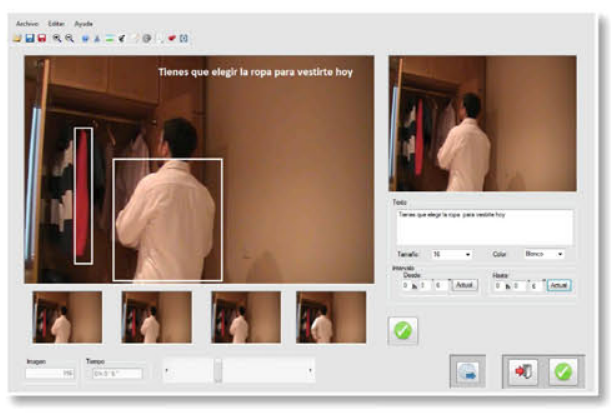

Fig. 1 AMELIE video editor

The current version of the AMELIE tool and video player are implemented in $\mathrm{C}++$ and $\mathrm{C \#}$ respectively, using Microsoft's (Redmon, WA) .Net Framework. OpenCV library is used to handle all video-based objects, as well as for the implementation of video-processing algorithms.

\section{Medical use cases}

\section{A. Minimally Invasive Surgery}

Minimally invasive surgery (MIS) has become a procedural standard for many surgical sub-specialties. These techniques forgo the traditional approach of open interventions, allowing surgeons to perform surgeries through minimal incisions in the patient's body. MIS interventions are less painful for the patient; have fewer post-operative complications associated, and can shorten hospital stays [4].

Surgeons approaching MIS must become proficient on a series of cognitive and technical skills particular to these surgeries. Moreover, educational processes are gradually being adapted from mentor-apprentice-based approaches towards structured, objective training and assessment programs, where direct involvement of residents in real surgeries is delayed until becoming proficient in the required skills. Several motivators can be identified behind this: social awareness on medical errors, the need to reduce costs in hospitals, or the overloaded schedules of surgeons [4].

Adoption of TEL for cognitive online training can be a useful way of optimizing education programs, allowing for anytime/anywhere training. Combined with the strengths provided by laparoscopic videos [5], e-learning in surgery is an effective way of breaking time, space, and cost barriers by offering online or blended education alternatives that are potentially more viable and feasible than on-site courses.

In this context, the AMELIE Authoring Tool provides the means to exploit the numerous raw OR-video repositories stored in hospitals to create augmented video-based structured didactic contents (Fig. 2). Contents may then be used as class material, or most importantly, uploaded to a TEL environment for online training. In general, the authoring tool enables content sharing, task reproducibility and adaptive and ubiquitous learning; thus, it contributes to the shortening of MIS cognitive skills' learning curves and optimizes the efficiency of training.

\section{B. Informal Carers}

Nowadays, the increasing population aging index makes customized care necessary to keep the quality of life of older adults at home. New models of care must be deployed, in which informal carers play a crucial role as one of the most important sources of care for older adults, suffering stress episodes, over-work and depression. The carer supervises the patient's daily activities, spends a lot of time with him and assists in the care process. For this reason, it is vital to provide the necessary support in order to help carers in this task and reduce their workload [6].

One of the main informal carers' requirements is the need of knowing how to manage particular elderly's assistance and their consequent problems [7]. The use of TEL can provide new approaches for carers to satisfy the growing demand for attendance and support. This learning approach will allow the informal carer to enhance the assistance provided, adapting and personalizing contents addressing cognitive, functional, behavioral and personality changes of their elderly relatives [8]. Video-based enhanced learning technologies provide informal carers with an interesting opportunity for the acquisition of assistance skills through an interactive and use-case based experience.

Employing AMELIE, several issues are being addressed: (1) creating personalized video-based contents considering carer's preferences and learning requirements as well as the assisted person's condition; (2) enhancing user experience by showing real use-case videos; (3) focusing on particular aspects of older people assistance by means of detailed videos with added information (Fig. 2); and (4) allowing carers to navigate through several assistance pathways thanks to the interactivity provided by the tool. 

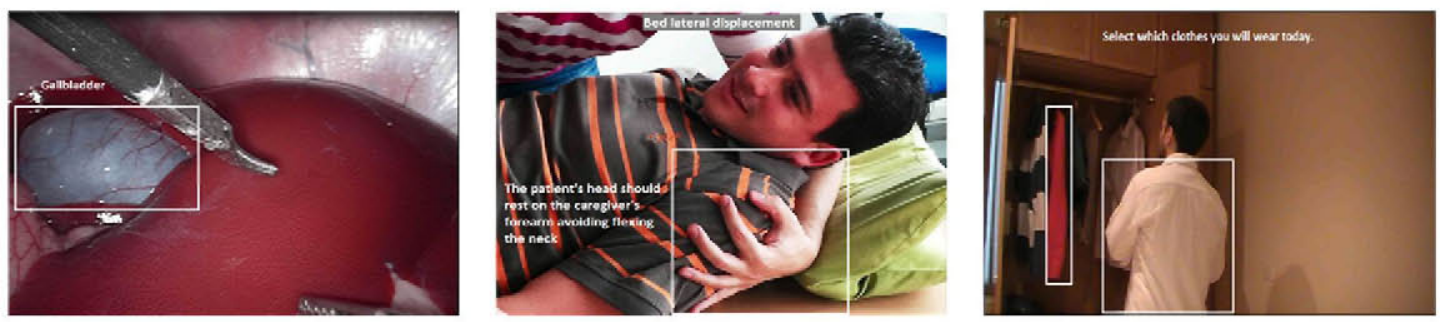

Fig. 2 Examples of augmented videos: Left: minimally invasive surgery; Middle: Informal carers; Right: Cognitive neurorehabilitation

\section{Cognitive Neurorehabilitation}

Acquired brain injury (ABI) is defined as a brain damage that suddenly and unexpectedly erupts in people's life. An $\mathrm{ABI}$ can be produced by different causes, such as stroke and traumatic brain injury. Associated cognitive impairments are the main cause of disability in developed societies [9]. Cognitive neurorehabilitation is the process whereby people with brain injury work together with health service professionals and others to remediate or alleviate said impairments. New technologies have led to higher intensity rehabilitation processes, extending therapies in an economically and sustainable way.

Some studies have proved that activity daily living (ADL) observation treatment is a good rehabilitative approach in stroke patients [10]. Additionally, the effectiveness of interactive videos (IV) has been verified on learning applications, where interactive dynamic visualization allows users to adapt the learning process to their individual cognitive skills [2][11]. IV refers to any video whose sequences and displayed information depend on the users' responses. Interactivity is provided by associating an interaction with any element tagged in the video scenes, modifying the video flow according to the way users interact with it.

Current research in this field is trying to ascertain whether IV is an appropriate technology to sustain personalized telerehabilitation processes. More specifically, the contemplated goal calls for patients to deal with real situations of daily living based on enhanced video-based environments [12]. In this context, AMELIE is used to define, tag and track the hot zones of the IV that will enable interactivity [13]. Using the enhanced video, the goal is to guide patients along every single scene (Fig. 2). Depending on their cognitive sequels, additional text, audio or virtual objects are inserted in order to help them reach the final goal. In this context, current work is focusing on adapting AMELIE's breakpoint inclusion functionality to set up the interactive videos and enable joining clips according to interactions and hot spots.

\section{USER VALIDATION}

The AMELIE tool has been validated in the context of project TELMA, which resulted in the creation of a TEL environment for MIS cognitive skills training [3]. The environment provided surgical trainees ubiquitous access to multimedia didactic units. These were created and uploaded by expert surgeons by means of AMELIE, based on enhanced laparoscopic videos.

Five surgeons (4 experts with teaching experience and 1 novice) performed a two-phase validation using AMELIE. The alpha test was performed under supervision of a developer. In the beta test supervision was avoided, and surgeons were allowed to freely use AMELIE for 30-60 minutes. A likert-type questionnaire was filled to obtain information regarding operational, functional and usability data.

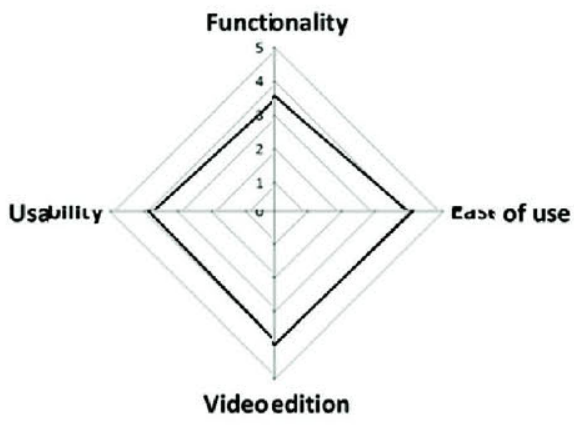

Fig. 3 Global results. (1: Low performance; 5: High performance)

Validation global results are shown in Fig. 3. Functionality of the tool, video edition tasks, ease of use and learning and usability were analyzed, in many cases showing good preliminary results. For a complete analysis of results the reader is referred to [3].

\section{Discussion}

In this work we have presented AMELIE, an authoring tool for video-based didactic content creation in e-health applications. Three real application scenarios have been 
described, each in a different stage of implementation and with a different target user.

A key advantage of AMELIE lies in its versatility. Creation of didactic contents is adaptable to any kind of clinical application; mostly related with training processes (medical professionals, informal carers), but also adjustable to telerehabilitation scenarios (patients). Moreover, the concept could be easily extended beyond the clinical world.

While AMELIE provides means to create complete contents combining multimedia information and assessment capabilities, its true kernel lies on its video editor. Proprietary solutions such as Adobe Premiere are usually aimed at professionals with background knowledge on video edition, and thus present a steep learning curve for non-experienced users. Additionally, the focus of their functionalities does not fall on edition for didactic purposes. AMELIE covers the gap by providing health professionals ways to easily enhance any video with didactic information.

Moreover, the tool can be adapted to different environments independently of their content presentation standard. Contents may be uploaded/downloaded directly to/from the e-learning platform of choice. For storage and retrieval purposes, multi-language documentation is handled via metadata fields generated in a relational data model through a predefined thesaurus. Integration of the video-player in the platform guarantees the correct content playback.

Depending on the final application, however, different approaches to its use may be contemplated. Deviations from the default scenario (creation of didactic units for e-learning applications) may be considered, for example with the definition of contents for classroom teaching, or the preparation of an IV for a telerehabilitation session.

Preliminary validation in MIS has yielded positive reception on its functionality and usability. While the rest of case uses described remain to show valid proof, these results encourage the usefulness of such tools in e-health applications. In order to corroborate them in diverse areas of medical specialties, new and larger validation studies are planned in the three application areas described in this work.

\section{vi. Conclusions}

The AMELIE tool can be a valuable asset for the creation of medical video-based contents in e-learning applications. The authoring tool's greatest strengths fall on its effective enhancement of videos from any source with didactic purposes and its versatility in any number of e-health applications. The tool is intuitive, easy to use and robust, and is accessible to any medical professional regardless of their computing knowledge.

\section{ACKNOWLEDGMENT}

Authors participate under the following research projects: MISTELA (LLP-Leonardo da Vinci-528125-LLP-1-20121-UK), CARECLOUD (INNPACTO IPT-2012-0599300000) and REHABILITA (CENIT-E CEN-20091043).

\section{REFERENCES}

1. Bloomfield JG, Jones A (2013) Using e-learning to support clinical skills acquisition: Exploring the experiences and perceptions of graduate first-year pre-registration nursing students - A mixed method study. Nurs Educ Today [Epub ahead of print]

2. Merkt M, Weigand S, Heier A et al (2011) Learning with videos vs. learning with print: The role of interactive features. Learn Instr, 21: 687-704.

3. Sánchez-González P, Burgos D, Oropesa I et al. TELMA: Technology enhanced learning environment for minimally invasive surgery (2012), J Surg Res, 182(1): 21-29.

4. Oropesa I, Sánchez-González P, Lamata P et al (2011) Methods and Tools for Objective Assessment of Psychomotor Skills in Laparoscopic Surgery. J Surg Res, 171: e81-e95.

5. Sánchez-González P, Cano AM, Oropesa I et al (2011) Laparoscopic Video Analysis for Training and Image Guided Surgery, Minim Invasive Ther Allied Technol, 20:311-320.

6. Ducharme FC, Levesque LL, Lachance LM et al (2011) Learning to Become a Family Caregiver' Efficacy of an Intervention Program for Caregivers Following Diagnosis of Dementia in a Relative. Gerontologist 51: 484-494.

7. Powell J, Chiu T, Eysenbach G (2008) A systematic review of networked technologies supporting carers of people with dementia, J Telemed Telecare 14: 154-156.

8. Powell J, Gunn L, Lowe P, et al (2010) New networked technologies and carers of people with dementia: an interview study. Ageing Soc 30: $1073-1088$.

9. World Health Organization. Burden of Disease Statistics; Available online: http://www.who.org/ (last access: april 2013).

10. Franceschini M, Agosti M, Cantagallo A et al (2010) Mirror neurons: actions observation treatment as a tool in stroke rehabilitation. Eur J Phys Rehab Med 46:517-523.

11. Martin M, Weigand S, Heier A et al (2011) Learning with videos vs. learning with print: The role of interactive features. Learn Instr 21: 687-704.

12. Martínez-Moreno JM, Solana J, Sánchez R, et al (2013) Monitoring visual attention on a neurorehabilitation environment based on Interactive Video. International Conference on Recent Advances in Neurorehabilitation: 182-185.

13. Luna M, Sánchez-González P, Bonilla E et al (2012) Detección y seguimiento de objetos en vídeos de actividades de vida diaria para rehabilitación de pacientes con daño cerebral adquirido. XXIX Annual Congress of the Spanish Society for Biomedical Engineering.

Corresponding author:

Author: Patricia Sánchez González

Institute: Biomedical Engineering and Telemedicine Centre, ETSI Telecomunicacíón, Universidad Politécnica de Madrid.

Street: Avda. Complutense, 30

City: Madrid

Country: Spain

Email: psanchez@gbt.tfo.upm.es 